\title{
The narrow mesh: A new shaped mesh for the treatment of inguinal hernia
}

\author{
Enrico Nicolo*
}

Emeritus, Department of Surgery, Jefferson Regional Medical Center, Pittsburgh, Pennsylvania, USA

Accepted: 22 July, 2020

Published: 23 July, 2020

${ }^{*}$ Corresponding author: Enrico Nicolo, MD, FACS, FICS Emeritus, Department of Surgery, Jefferson Regional Medical Center, Pittsburgh, Pennsylvania, USA, Tel: 4127203038; E-mail: enicolo@aol.com

Keywords: Inguinal hernia; Alloplastic mesh; Hernia recurrence; Chronic inguinal pain; Inguinodynia

https://www.peertechz.com

\section{Check for updates}

\begin{abstract}
The present communication relates to inguinal hernia repair, and more particularly to a preshaped inguinal hernia prosthetic material, polypropylene, $6 \times 2.5$ centimeters in size, (Figure 1) that has a lateral semicircular non-encircling cord locating structure in order to protect the spermatic cord and that it is interposed between the conjoined tendon and the inguinal ligament in such a way as to repair the hernia and at the same time to reconstitute the physiology of the inguinal canal.
\end{abstract}

\section{Introduction}

Inguinal hernia repair remains one of the most frequent procedures in general surgery, with some 20 million operations being performed worldwide each year. The prosthetic repair is now accepted worldwide as the gold standard in inguinal hernia repair [1]. Several studies claim its superiority over suture repair especially in cases of recurrent hernias. The mesh repair essentially bridges to cover the gap forming the hernia defect, without stretching patient's tissues over the defect, thus allowing tissues to remain 'tension-free'. On one hand the use of mesh in the inguinal hernia repair substantially reduced the recurrence rate and the rehabilitation period as compared to sutured repairs. On the other hand, the mesh brought with itself several complications, such as protrusion, extrusion, infection, intestinal fistulization, and especially chronic inguinodynia $[2,3]$.

Furthermore the mesh, once in place, is rigid, passive, adynamic, and aphysiological, addressing only the anatomical aspect of the posterior wall of the inguinal canal without restoring its function.

In the attempt of restoring not only the strength of the posterior wall of the inguinal canal but also its physiological properties of active contraction and passive relaxation, we propose a new alloplastic mesh, called narrow mesh (NM), $6 \times 2.5 \mathrm{~cm}$. in size, that is interposed between the conjoined tendon and the inguinal ligament and shaped in such a way to accommodate the spermatic cord without encircling it and (Figure 1).

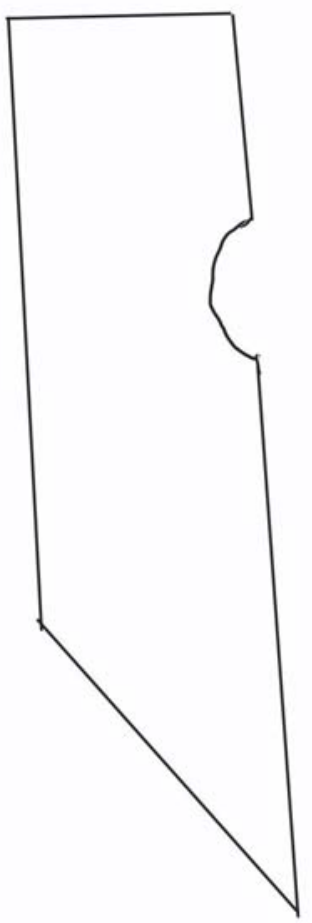

Figure 1: A Lateral non- encircling cord locating structure to protect the spermatic cord.

Citation: Nicolo E (2020) The narrow mesh: A new shaped mesh for the treatment of inguinal hernia. J Surg Surgical Res 6(2): 120-122. 


\section{Ibrid operation}

Ibrid operation consists of the use of pure tissue (modified Bassini) and NM.

The NM is interposed between the inguinal ligament and the conjoint tendon.

\section{Operative technique}

The lateral border of the narrow mesh is sutured in a continuous fashion to the native inguinal ligament starting medially at its origin on the pubic spine and proceeding upward and laterally for $2-3 \mathrm{~cm}$ beyond the contact of the internal oblique muscle with the inguinal ligament. After this, the conjoint tendon is approximated to the medial border of the narrow mesh (considered the new inguinal ligament) in a continuous fashion; starting medially, at the pubic spine, the first two stitches include also the lateral border of the rectus muscle; then proceeding upwardly the conjoint tendon and the internal oblique muscle are sutured to the medial border of the narrow mesh. The last stitch is aimed to the reconstruction of the internal inguinal ring. When this suture is tied, the newly reconstructed internal inguinal ring will be calibrated, with the spermatic cord taking an oblique course surrounded superiorly and medially by the conjoint tendon.

\section{Discussion}

Edoardo Bassini [4], in 1889 posted a milestone in the history of hernia surgery, reporting in his monograph, a series of 262 inguinal hernias operated with his original operative method, with only 7 recurrences ( $2.6 \%)$. Bassini's operation [5-9], epitomizes the essential steps for an ideal tissue inguinal hernia repair whose cornerstone is the opening of the transversalis fascia and the mobilization of the triple layer (internal oblique muscle, transversus muscle, and transversalis fascia), so that this triple layer may be reappoximate to the isolated posterior border of the inguinal ligament, without tension, reconstructing the posterior wall of the inguinal canal and the internal inguinal ring. This is to restore not only the anatomy but also the physiology of the inguinal canal.

Unfortunately Bassini's principles of hernia repair were lost in time and in a tangle of a honest misunderstanding and misinterpretation that obscured the original teachings. Many surgeons abandoned the opening of the transversalis fascia adopting their own modifications of the original operation of Bassini. Results of the short cut Bassini's operation were unsatisfactory, and none of the modifications achieved better results than the original operation of Bassini. Without opening the transversalis fascia and without the mobilization of the triple layer, they transformed the tensionless original operation of Bassini in a 'tension' operation with a higher recurrence rate up to $25 \%$, an embarrassment for the surgical community.

The vacuum was filled in the early 80 's by Lichtenstein [1], who developed a tension free inguinal hernia repair using a Polypropylene (PP) mesh to bridge the gap forming the hernia defect. In this way the patient's tissues are not 'stretched' over the defect, thus allowing the tissue to remain tension-free.
Recurrences were drastically reduced but a foreign body was inserted into the inguinal canal and the necessity of creating a key-hole to accomodate the spermatic cord caused a constriction of the spermatic cord itself.

Moreover literature data showed that, despite a low learning curve, surgeons were far from reaching the good results reported by the Lichtenstein [1] and continued to experience high recurrences.

To overcome the problem, surgeons demanded for larger and always more complex meshes in the erroneous judgment that a such a mesh could provide further strength to the repair.

Recent studies show that this attitude did not change the rate of recurrence at all.

For this reason many Authors are beginning to question the real efficacy of the mesh repair, especially at the light of the high incidence of chronic inguinal pain $[2,3]$.

The pure tissue repair has in fact the advantage of restoring the physiology of the inguinal canal with only a little increase in the recurrence rate, and the advantage of no putting any foreign body in the inguinal canal, thus reducing the meshrelated complications, especially postoperative chronic inguinodynia.

In order to maintain the advantages of both techniques, the modified Bassini operation and mesh repair according to Lichtenstein, we created an improved implantable inguinal mesh (Figure 1) $6 \times 2.5$ centimeters in size having a lateral nonencircling cord locating structure to protect the spermatic cord and it is configured in such a way as to reconstitute the physiologic structure of the inguinal canal into the prehernia state.

Such a configuration and structure allow the use of patient's original tissues to protect the spermatic cord structures from damage, so that both, the NM and the patient's tissues, may achieve the complete cure of the inguinal hernia and the reconstitution of the pre-hernia physiology. The NM, due to its innovative shape, has in fact two complementary actions in repairing the inguinal hernia.

First, it allows the native tissues (conjoint tendon) to be repositioned mostly at the original anatomical location so that the physiology of the inguinal canal is restored, and second reduces the tension on the suture line simply by increasing the height of the inguinal ligament in order to make easier the coaptation of the conjoint tendon to the neo-inguinal ligament that is the medial border of the narrow mesh.

Furthermore, the reduced dimension and volume of the mesh and the absence of the key hole encircling the spermatic cord are a protection factor for the cord, that will not be strangulated by its surrounding mesh with its fibrous transformation.

Essentially, the use of the NM may be considered as a modification of the modified Bassini operation, the so-called North American Bassini, with the interposition of the NM 
between the conjoined tendon and the inguinal ligament. In fact the primary aim of the repair is the creation of a neoinguinal ligament that is moved medially as much as the width of the mesh itself, about 2.5 centimeters. The medial aspect of the narrow mesh can be considered the new inguinal ligament. This will allow the approximation of the conjoint tendon to the neo-inguinal ligament, without any tension. The remodeling of the internal inguinal ring and the creation of a reinforced posterior wall is so achieved physiologically by the viable tissues, the conjoint tendon. This confirms the fact that it is not the foreign body that prevents recurrences but the restoring of the physiology of the inguinal canal structures.

The regaining of a more physiological behavior and the use of a small sized mesh could be both protective factors against the development of chronic inguinal pain. Furthermore the NM, due to its small size, avoids the long-term complications due to shrinking of the mesh, especially chronic inguinodynia, caused by the presence of a large piece of mesh into the inguinal canal.

The Narrow Mesh, interposed between the inguinal ligament and the conjoint tendon, reduces the tension on the suture line by advancing medially the inguinal ligament.

The newly reconstructed posterior wall of the inguinal canal and the internal inguinal ring are formed by muscles, the conjoint tendon, so that the physiology of the inguinal canal, the shutter mechanism, is restored.

As the physiology is restored, the cure is to be expected.

The small in size NM does not encircle the spermatic cord, it does not bridge the defect but it approximates tissues.

It protects the spermatic cord and it is instrumental in preventing chronic inguinodynia.

The shrinking of the mesh, up to $60 \%$, is not a disadvantage in this case, but it has the advantage of further approximation of the conjoint tendon to the native inguinal ligament preserving and enhancing the physiology of the shutter mechanism.

\section{Conclusion}

Clinical studies are warranted to evaluate the proposed claims of efficacy and safety and the prospective of a better outcome of the use of the Narrow Mesh (NM) for the repair of inguinal hernia.

\section{References}

1. Lichtenstein IL, Shulman AG, Amid PK, Montllor MM (1989) The tension-free hernioplasty. Am J Surg 157: 188-193. Link: https://bit.ly/3eTdvMi

2. Klinge $U$, Krones CJ (2005) Can we be sure that the meshes do improve the recurrence rates? Hernia 9: 1-2. Link: https://bit.ly/3jrVbgJ

3. Fischer JE (2013) Hernia repair: why do we continue to perform mesh repair in the face of the human toll of inguinodynia? Am J Surg 206: 619-623. Link: https://bit.ly/3fQWHqK

4. Bassini E (1889) Nuovo metodo operativo per la cura radicale dell'ernia inguinale. Prosperini, Padova.

5. Nicolo $E$ (2009) Bassini landmark procedure for the radical cure of inguinal hernia. Ciné-Med Publishing. Link: https://bit.ly/3eQs9DW

6. Bendavid R (2012) The Shouldice Hospital repair in hernia. In: Jones D Fischer JE, editors. Master techniques in surgery: hernia. 6th ed. Philadelphia: Lippincott Williams \& Wilkins 71-86.

7. Amid PK, Lichtenstein IL (1998) Long-term results and current status of the Lichtenstein open tension-free hernioplasty. Hernia 2: 89-94. Link: https://bit.ly/3fPVsYP

8. Amid PK (2003) The Lichtenstein repair in 2002: an overview of causes of recurrence after Lichtenstein tension-free hernioplasty. Hernia 7: 13-16. Link: https://bit.ly/3eQnqT5

9. Neumayer L, Giobbie-Hurder A, Jonasson O, Fitzgibbons R, Dunlop D, et al (2004) Open mesh versus laparoscopic mesh repair of inguinal hernia. N Engl J Med 350: 1819-1827. Link: https://bit.ly/20JxUJ0

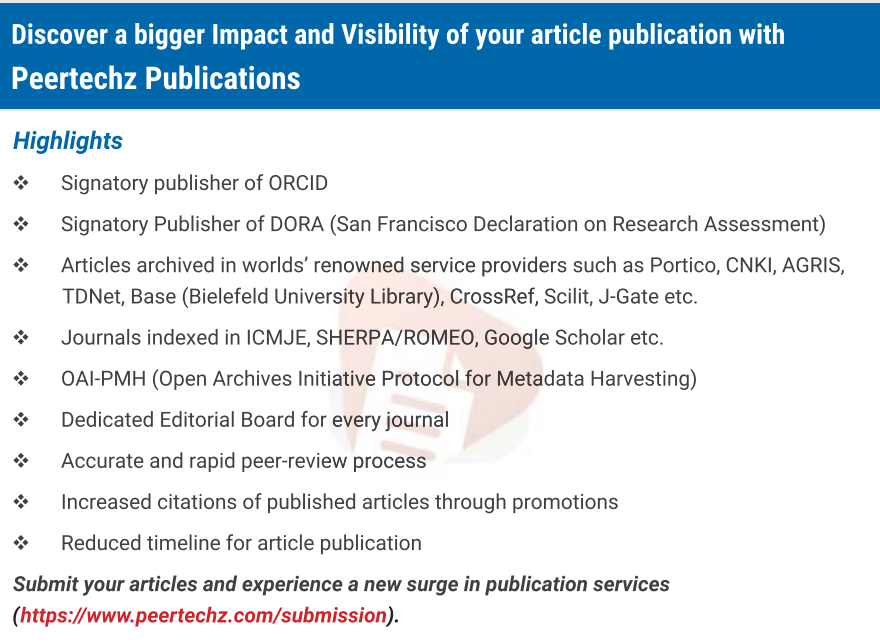

Copyright: (c) 2020 Nicolo E. This is an open-access article distributed under the terms of the Creative Commons Attribution License, which permits unrestricted use distribution, and reproduction in any medium, provided the original author and source are credited. 